\title{
Effect of a chicken comb extract-containing supplement on subclinical joint pain in collegiate soccer players
}

\author{
MASAFUMI YOSHIMURA $^{1}$, YOSHIHIRO AOBA ${ }^{1}$, KIYOHITO NAITO $^{2}$, \\ TAIJI WATARI ${ }^{2}$, SAYA MURAKAMI $^{3}$, KAORI YOSHIMURA $^{4}$, TAKESHI NAKAGAWA ${ }^{4}$, \\ TETSURO YAMAMOTO $^{4}$, HIDEYO YAMAGUCHI ${ }^{4}$ and ISAO NAGAOKA $^{5}$ \\ ${ }^{1}$ Juntendo University Graduate School of Health and Sports Science, Inzai, Chiba; \\ ${ }^{2}$ Department of Medicine for Motor Organ, Juntendo University Graduate School of Medicine, Tokyo; \\ ${ }^{3}$ Everlife Co., Ltd., Fukuoka; ${ }^{4}$ Total Technological Consultant Co., Ltd.; ${ }^{5}$ Department of Host Defense \\ and Biochemical Research, Juntendo University Graduate School of Medicine, Tokyo, Japan
}

Received October 4, 2011; Accepted December 9, 2011

DOI: 10.3892/etm.2011.431

\begin{abstract}
Much of our focus of attention has been on subclinical or subtle joint pain experienced by healthy soccer players. The present study aimed to determine at which joint such subclinical pains are the most prominent, and to examine the pain-relieving effect of a chicken comb extract (CCE)-containing supplement product (test product) on these athletes. A total of 46 collegiate soccer players, consisting of 24 leading and 22 substitute players, belonging to a university soccer team were enrolled for measuring the pains at 4 different joints (ankle, knee, hip and shoulder) using 3 pain subscales of a 100-mm visual analog scale (VAS) ('pain at rest', 'pain on pressing' and 'pain on moving'), and participated in a prospective, double-blind, controlled study. A total of 23 subjects each received the test product $(4,800 \mathrm{mg} /$ day $)$ (test group) and placebo (placebo group) for 12 weeks. VAS pain scores of individual joints were evaluated at baseline and following 4, 8 and 12 weeks of the intervention. VAS scores for the 'pain on moving' subscale in 46 enrolled subjects were highest at the ankle joint, and thus the values (abbreviated as 'pain scores') were used as a parameter for efficacy assessment of the test product. Compared to the baseline, the pain scores were significantly decreased for the dominant foot (but not for the non-dominant foot) in the total subpopulation (at week $4 ; \mathrm{p}<0.01$ ) and the leading player subpopulation (at week 4; $\mathrm{p}<0.01$ and at week $12 ; \mathrm{p}<0.05)$ in the test group $(\mathrm{n}=19$ and 11 , respectively). In comparison between the test product and placebo groups, the pain scores were significantly changed for
\end{abstract}

Correspondence to: Professor Isao Nagaoka, Department of Host Defense and Biochemical Research, Juntendo University Graduate School of Medicine, 2-1-1 Hongo, Bunkyo-ku, 113-8421 Tokyo, Japan E-mail: nagaokai@juntendo.ac.jp

Key words: hyaluronan, soccer player, joint pain, supplementary diet, athlete the dominant foot $(\mathrm{p}<0.05)$ at week 4 in the total subpopulation and at week 12 in the leading player subpopulation in the test group. Thus, subclinical joint pain is most prominently observed at the ankle joint of the dominant foot in healthy young soccer players and may be improved by the daily intake of the CCE-containing supplement.

\section{Introduction}

Soccer is one of the sports most likely to cause not only clinically recognized joint injuries but also unrecognized minute ones due to the overuse, repetitive impact and torsional loading of joints. Furthermore, these conditions may be aggravated by several factors, including excessive mechanical loads and repeated microtrauma. These factors are known to enhance articular cartilage damage in soccer players. In this context, there are supporting data demonstrating that the risk of cartilage damage is increased in soccer (and certain other sports), where the repetitive intense impact and torsional loading are subjected to the joints (1), and may be implicated in the chronic development of osteoarthritis (OA) (2-4). Indeed, several clinical studies have demonstrated that the risk of OA is higher in soccer players, as well as in certain other groups of athletes, than in control non-athlete groups (5-11).

In the course of health management of collegiate soccer players, we noticed that most players, particularly leading players, suffer from subtle lower extremity joint pains that are self-recognized only when they engage in the training and/or soccer games, although they are not diagnosed as having joint injuries. Thus, we speculated that these conditions may result from clinically unrecognized minute joint injuries and that such injuries may also increase the risk of subsequent development of OA or other degenerative joint diseases (1). The aim of the present study was to examine at which joints the minute injury-mediated subclinical joint pains occur and are observed in soccer players. For this purpose, pains were measured at several different joints, mainly lower extremity joints, using 3 pain subscales of a $100-\mathrm{mm}$ visual analog scale (VAS) in a team of collegiate soccer players. 
In addition, we focused on the potential of nutraceuticals for the prevention and treatment of such subclinical joint pains. Glucosamine and chondroitin are two popular dietary supplements being promoted for the maintenance of joint health among athletes (12-15). In addition, our previous study demonstrated that the intake of a dietary supplement containing chicken comb extract (CCE) rich in hyaluronan (HA) (CCE supplement) was effective in improving pain and dysfunction of afflicted joints in patients with knee OA (16). Based on these data, we conducted a prospective, double-blind, controlled study, in which the effect of the CCE supplement on joint pain was evaluated in a team of collegiate soccer players without any previously diagnosed joint injury.

\section{Materials and methods}

Subjects. The present study was approved by the Human Experimentation Ethics Committee of Juntendo University (Japan) and was conducted in accordance with the principles of the amended Declaration of Helsinki and 'Ethical Guidelines for Epidemiological Research' (established by the Japanese Government in 2004). Written informed consent was obtained from all subjects prior to their enrollment in the study. The enrolled subjects belonged to a soccer team of Juntendo University School of Health and Sports Science, and included both leading and substitute players.

Exclusion criteria were: clinically recognized joint injuries; common use of health food or cosmetics containing constituents with analgesic and/or chondroprotective potential (e.g., glucosamine, chondroitin and HA); known allergy to certain constituent(s) or material(s) of the test supplement; previous or current (within the previous 3 months) treatment or planned treatment to be administered during the study period with intra-articular injections of HA or corticosteroids; routine intake of anti-resorptive drugs, including bisphosphonates or estrogen; participation in another clinical study and the presence of any medical condition or history judged by the medical investigator to preclude the subject's inclusion in the study.

Among the eligible subjects, appreciable differences in the amount of soccer-related activities were observed between leading and substitute players. According to the estimation by the team leader and coaches, the average amount of physical activities in the training and soccer games for the leading players during the study period was 1.4 - to 1.7-times greater than that for the substitute players. The amount of soccer-related activities also varied based on the players' position within the team or on the pitch, being the highest in midfielders and the lowest in goalkeepers. Taking these points into consideration, enrolled subjects were divided into 2 groups so that they were distributed evenly in terms of their playing positions, as well as leading and substitute players.

Intervention and subject group assignment. The test product was a commercially available $300-\mathrm{mg}$ capsule-form product (Kojun $^{\circledR}$ ), containing $157.5 \mathrm{mg}$ of CCE, of which approximately $4.5 \mathrm{mg}$ was HA, together with $20 \mathrm{mg}$ of calcium lactate, $10 \mathrm{mg}$ of propolis extract, $4.9 \mathrm{mg}$ of chitosan oligosaccharide, $5.0 \mathrm{mg}$ each of vitamins $B_{1}$ and $B_{6}, 2.5 \mathrm{mg}$ of vitamin $E, 2.0 \mathrm{mg}$ of ferric pyrophosphate, $0.1 \mathrm{mg}$ of vitamin $\mathrm{B}_{12}$ and $192.5 \mathrm{mg}$ of the vehicle (comprising of crystalline cellulose, dextrin and fatty acid sugar esters). Subjects were assigned to receive 16 capsules per day of the test product (test group) or 16 capsules per day of the 'dummy' placebo, containing only the vehicle (placebo group). All subjects were instructed to take allocated capsules in a dose of 8 capsules twice daily following breakfast and dinner with the aid of a sufficient amount of drinking water for a consecutive 12 weeks. The study was performed during the 2009 summer-fall soccer competition season. Adherence to the intervention was assessed on the basis of consumption recorded in the self-administered study diary, and a value $<85 \%$ was considered to constitute a protocol violation.

Procedures. All subjects underwent clinical and laboratory examinations at baseline and at weeks 4,8 and 12 following the commencement of intervention to follow the changes in pain levels during the 12-week intervention period. Due to the characteristics of soccer, the lower extremity joints are expected to be at a greater risk of suffering repetitive impact and torsional loading than the upper extremity joints. On the basis of this, we selected the 3 lower extremity joints (i.e., ankle, knee and hip joints) as the main target joints, together with a shoulder joint as a representative of upper extremity joints, for evaluating the pain-relieving effect of the test product.

Pain levels of the individual test joints were measured using the self-diagnosed 3 pain subscales of the 100-mm VAS: 'pain at rest', 'pain on pressing' and 'pain on moving'. Each VAS pain subscale was scored from 1 to 100 , where 0 indicated no pain and 100 indicated the most intense pain ever experienced.

Tolerability and safety was assessed throughout the study on the basis of the incidence and severity of intervention-related adverse events (side-effects) as well as abnormal changes in blood pressure, pulse rate and laboratory tests, including hematology, biochemical profile and urinalysis.

Statistical analysis. Values of all variables were expressed as the means \pm standard deviation (SD). Individual VAS scores for 3 pain subscales at 4 different joints measured prior to the start of intervention were compared by the Student's t-test. Baseline characteristics of the total subject population and the leading players were compared between the test and placebo groups by the Student's t-test for continuous variables and by the Mann-Whitney U test for category variables. Pain scores during the intervention were compared to the baseline values by the Student's t-test. Comparisons of the changes from the baseline in pain scores were also performed by the Student's t-test. A p-value $<0.05$ was considered to indicate a statistically significant difference.

\section{Results}

Comparison of VAS scores for 3 pain subscales among 4 test joints. The severities of pain at 4 different joints (e.g., ankle, knee, hip and shoulder joints), as measured by the use of 3 pain subscales of a 100-mm VAS, were compared using 46 enrolled collegiate soccer players, consisting of 24 leading and 22 substitute players, prior to the commencement of intervention. As shown in Fig. 1, the total population analysis with all the subjects indicated that the 'pain on moving' subscale scores were higher than the 'pain at rest' and 'pain on pressing' subscale scores at all of the 4 test joints, and that the values 
A

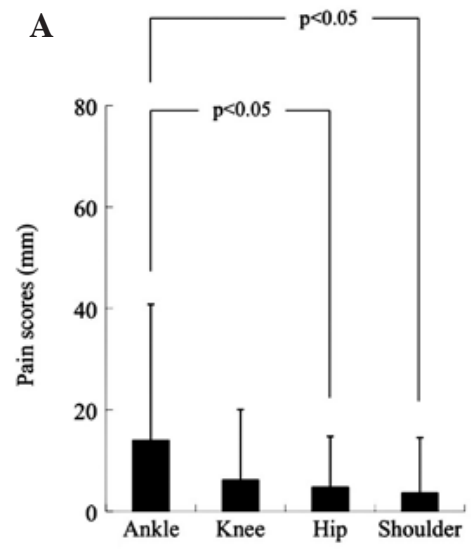

D

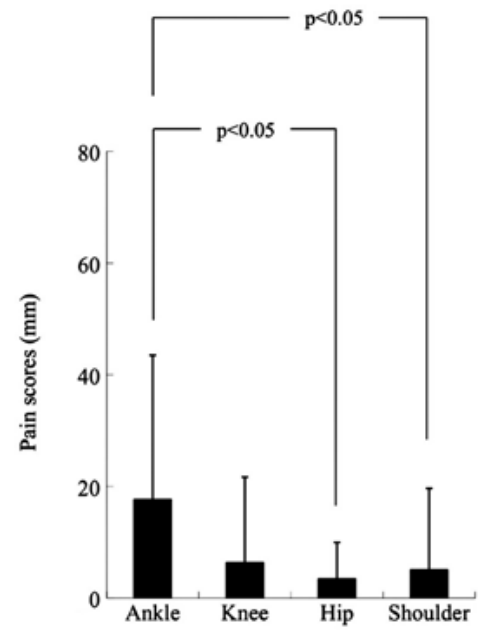

B

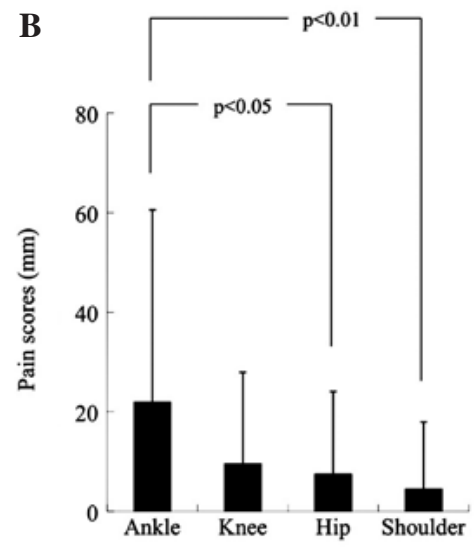

$\mathbf{E}$

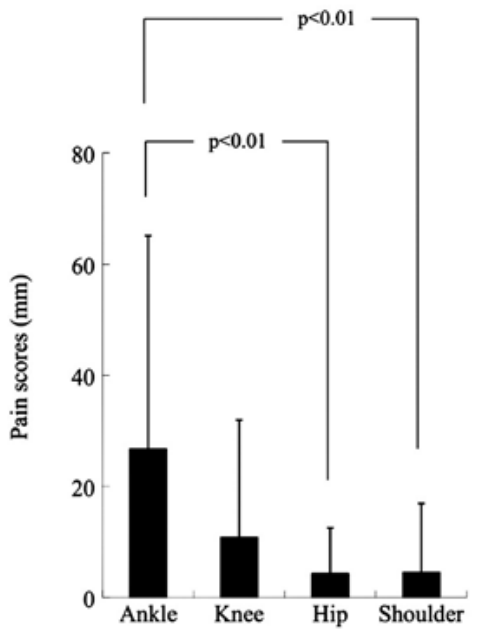

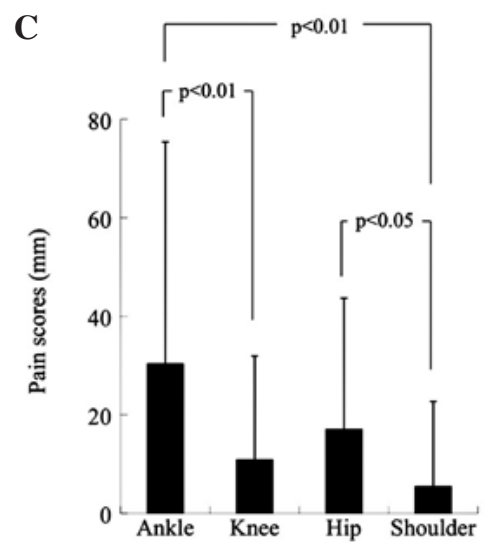

$\mathbf{F}$

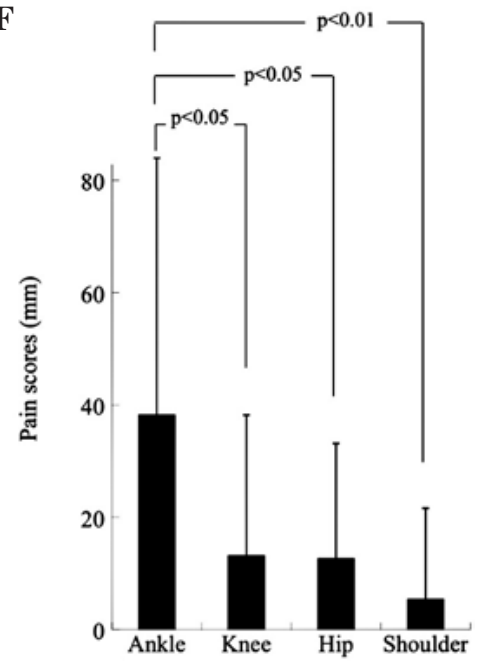

Figure 1. Comparison of VAS scores for the 3 pain subscales in the 4 different joints of the total subpopulation and the leading player subpopulation. VAS scores for the 3 pain subscales. (A and D) 'pain at rest', (B and E) 'pain on pressing' and (C and F) 'pain on moving' in the 4 different joints (ankle, knee, hip and shoulder joints) of the total subpopulation ( $\mathrm{n}=46 ; \mathrm{A}, \mathrm{B}$ and $\mathrm{C})$ and the leading player subpopulation $(\mathrm{n}=24 ; \mathrm{D}, \mathrm{E}$ and F). VAS, visual analog scale.

at the ankle joint were the highest and those at the shoulder joint were the lowest among the 4 joints in the 3 pain subscale scores (Fig. 1A-C). Almost the same results were obtained in the subpopulation analysis with the lead players (Fig. 1D-F). However, no such clear results were obtained with the substitute players, probably due to the fact that the VAS scores for every pain subscale were too low in the substitute player group to evaluate reliably. The ankle joint VAS scores for 'pain at rest' and 'pain on moving' subscales were $14.0 \pm 26.8$ and $30.3 \pm 45.1(\mathrm{p}=0.037)$ in the total subject population, $17.7 \pm 25.8$ and $38.2 \pm 45.8(\mathrm{p}=0.062)$ in the leading player subpopulation, and $9.9 \pm 27.9$ and $21.8 \pm 43.7(\mathrm{p}=0.290)$ in the substitute player subpopulation, respectively.

Based on all these data, the VAS scores for 'pain on moving' at the ankle joint (hereafter presented as 'pain scores') were utilized as the parameter for the evaluation of the efficacy of the test product, and the total subject population and leading player subpopulation were subjected to efficacy analyses.

Characterization of study groups. Of the 46 enrolled subjects, 23 were assigned to the test product group and placebo group. A total of 4 of the subjects in the 2 groups withdrew from or discontinued the study during the intervention period for a number of reasons, including hospitalization due to bone or joint injuries, trauma and other medical problems, and withdrawal from the soccer team. Thus, 38 subjects (19 each in both the test and placebo groups) fulfilled the eligibility criteria, completed the study and were analyzed for the efficacy assessment. The eligible subjects in the test and placebo groups comprised 11 and 10 leading players and 8 and 9 substitute players, respectively.

As shown in Table I, the demographic and physiological characteristics (age, height, weight, body mass index, blood pressure and pulse rate) did not differ between the test and placebo groups. The number of leading and substitute players and the distribution of the position of players was well balanced between the 2 groups.

Regarding the pain scores, we analyzed the dominant foot and the non-dominant foot separately, since the extent of joint loads and the risk of joint injury were considered somewhat different between the dominant and non-dominant feet. The average pain scores for the dominant foot were slightly higher than those for the non-dominant foot (Table I).

Efficacy of the test supplement. Table II shows the changes in the pain scores analyzed with the total subject population and the leading player subpopulation in the test and placebo groups. Pain scores of the dominant and non-dominant feet 
Table I. Baseline data of eligible subjects in the test and placebo groups who completed the study.

\begin{tabular}{|c|c|c|c|}
\hline Variables $^{\mathrm{a}}$ & Test group $(n=19)$ & Placebo group $(n=19)$ & p-value \\
\hline Age (years) & $19.5 \pm 1.2$ & $19.7 \pm 1.3$ & 0.521 \\
\hline Height (cm) & $172.7 \pm 5.7$ & $172.2 \pm 6.2$ & 0.802 \\
\hline Weight (kg) & $66.05 \pm 5.65$ & $66.45 \pm 6.52$ & 0.843 \\
\hline Body mass index $\left(\mathrm{kg} / \mathrm{m}^{2}\right)$ & $22.14 \pm 1.32$ & $22.38 \pm 1.24$ & 0.579 \\
\hline Systolic blood pressure (mmHg) & $110.9 \pm 9.0$ & $116.4 \pm 8.9$ & 0.068 \\
\hline Diastolic blood pressure (mmHg) & $61.5 \pm 8.4$ & $62.7 \pm 6.3$ & 0.618 \\
\hline Pulse rate (beats/min) & $53.9 \pm 7.0$ & $53.5 \pm 6.9$ & 0.871 \\
\hline $\begin{array}{l}\text { Leading players/substitute players } \\
\text { (Number of subjects) }\end{array}$ & $11 / 8$ & $10 / 9$ & 0.795 \\
\hline $\begin{array}{l}\text { Distribution of the position of the players } \\
\text { (Number of subjects) MF/DF/FW/GK }\end{array}$ & $10 / 6 / 1 / 2$ & $9 / 6 / 4 / 0$ & 0.246 \\
\hline \multicolumn{4}{|l|}{ Pain scores of the ankle joints } \\
\hline Dominant foot & $22.29 \pm 23.72$ & $17.01 \pm 27.58$ & 0.531 \\
\hline Non-dominant foot & $17.96 \pm 24.17$ & $15.32 \pm 26.56$ & 0.750 \\
\hline
\end{tabular}

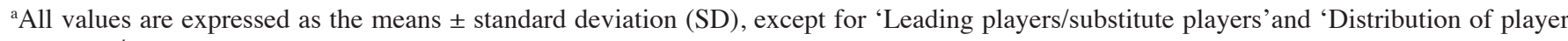
position'. ' ${ }^{\mathrm{MF}}$, midfielder; DF, defender; FW, forward; GK, goalkeeper.

Table II. Changes in the pain scores for the dominant and non-dominant feet during the 12 -week intervention period in the test and placebo groups.

\begin{tabular}{|c|c|c|c|c|c|c|}
\hline \multirow[b]{2}{*}{ Subjects } & \multirow[b]{2}{*}{ Group } & \multirow{2}{*}{$\begin{array}{l}\text { Foot, dominant } \\
\text { or non-dominant }\end{array}$} & \multicolumn{4}{|c|}{ Pain scores $(\mathrm{mm})^{\mathrm{a}}$} \\
\hline & & & Baseline & Week 4 & Week 8 & Week 12 \\
\hline \multirow{4}{*}{$\begin{array}{l}\text { Total population } \\
(\mathrm{n}=38)\end{array}$} & Test $(n=19)$ & Dominant & $22.29 \pm 23.72$ & $8.92 \pm 15.39^{b}$ & $14.09 \pm 18.57$ & $12.05 \pm 17.11$ \\
\hline & & Non-dominant & $17.96 \pm 24.17$ & $18.55 \pm 25.11$ & $17.55 \pm 19.86$ & $15.55 \pm 17.70$ \\
\hline & Placebo $(n=19)$ & Dominant & $17.01 \pm 27.58$ & $15.93 \pm 22.14$ & $16.11 \pm 26.40$ & $13.32 \pm 17.50$ \\
\hline & & Non-dominant & $15.32 \pm 26.56$ & $23.61 \pm 30.84$ & $12.57 \pm 23.01$ & $13.69 \pm 17.48$ \\
\hline \multirow{4}{*}{$\begin{array}{l}\text { First-line players } \\
(\mathrm{n}=21)\end{array}$} & Test $(n=11)$ & Dominant & $29.91 \pm 23.68$ & $13.72 \pm 18.90^{b}$ & $18.51 \pm 21.92$ & $7.86 \pm 12.26^{c}$ \\
\hline & & Non-dominant & $19.70 \pm 22.69$ & $19.20 \pm 26.27$ & $21.01 \pm 20.95$ & $12.88 \pm 11.89$ \\
\hline & Placebo $(n=10)$ & Dominant & $19.93 \pm 27.63$ & $20.26 \pm 22.76$ & $24.29 \pm 32.48$ & $18.79 \pm 21.04$ \\
\hline & & Non-dominant & $16.85 \pm 26.05$ & $28.35 \pm 36.99$ & $14.36 \pm 27.41$ & $14.50 \pm 18.73$ \\
\hline
\end{tabular}

aValues are expressed as the means \pm standard deviation $(\mathrm{SD}) ;{ }^{\mathrm{b}} \mathrm{p}<0.05 ;{ }^{\mathrm{c}} \mathrm{p}<0.01$ against the baseline (paired Student's t-test).

are presented separately. Significant reductions in the pain scores were observed only for the dominant foot (but not for the non-dominant foot) in the test group (but not in the placebo group); at week $4(\mathrm{p}<0.05)$ in the total population and at weeks $4(\mathrm{p}<0.05)$ and $12(\mathrm{p}<0.01)$ in the leading player subpopulation.

Fig. 2 shows the changes from baseline in the pain scores for the dominant and non-dominant feet of the total population and leading players in the 2 groups. Of note, the pain scores were significantly reduced in the dominant foot of the test group compared to the placebo group at week $4(\mathrm{p}<0.05)$ in the total population (Fig. 2A) and at week $12(\mathrm{p}<0.05)$ in the leading player subpopulation (Fig. 2C). By contrast, the pain scores were not significantly different between the test and placebo group for the non-dominant foot in the total population (Fig. 2B) and the leading player subpopulation (Fig. 2D).
Safety of the test supplement. A total of 11 subjects of the test group $(n=23)$ and 12 subjects of the placebo group $(n=23)$ reported at least 1 adverse event, but not for injuries and trauma during the intervention period. The total number of reported adverse events was 26 in the test group and 21 in the placebo group, and there was no significant difference in the frequency between the 2 groups. Individual adverse events and their frequencies in the test group compared to the placebo group were: upper respiratory infections (20 vs. 17); rhinitis (0 vs. 2); diarrhea (2 vs. 0); dehydration (1 vs. 0); retinal detachment (0 vs. 1); urticaria (0 vs. 1$)$; herpes ( 1 vs. 0 ); anemia ( 1 vs. 0 ) and pneumonia (1 vs. 0). All adverse events were judged by the medical investigator to be unrelated to the intervention. In the 2 groups, measurements of physiological parameters (weight, body mass index, blood pressure and pulse rate) and routine laboratory tests (hematology, blood 

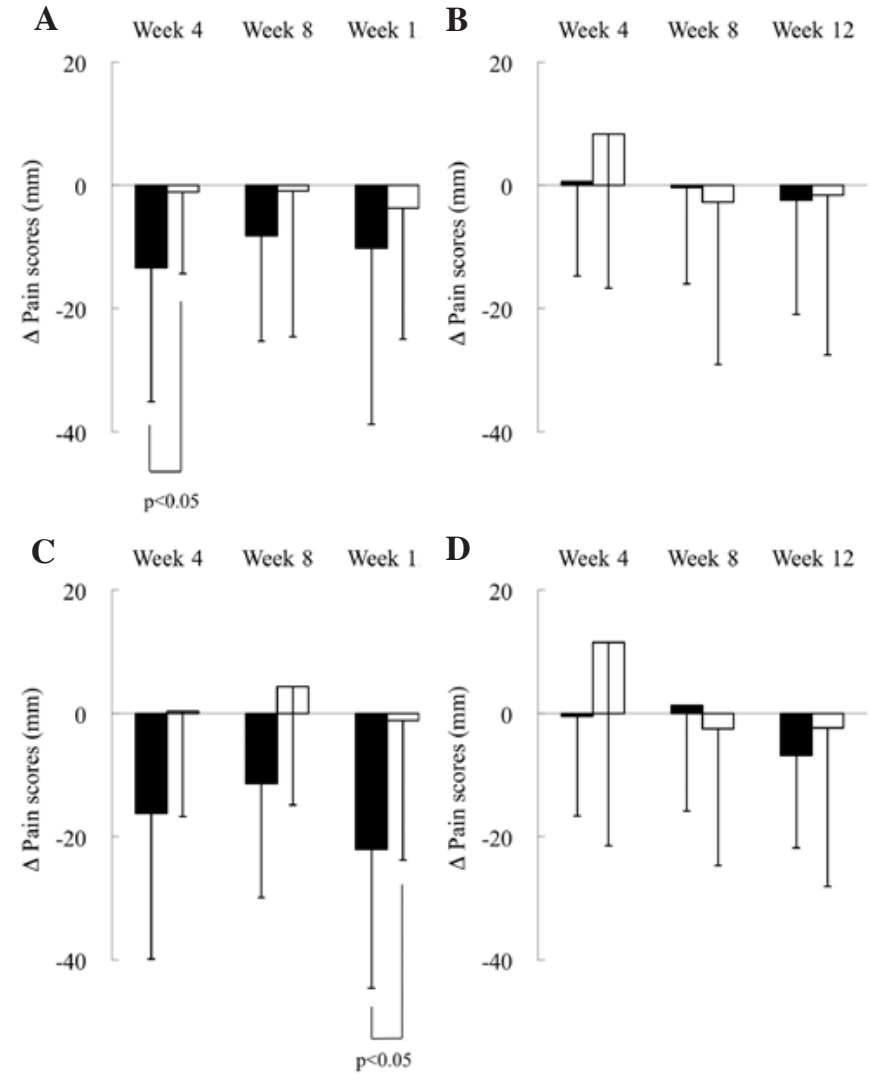

Figure 2. Changes from the baseline in the pain scores for the dominant and non-dominant feet in the test and placebo groups during the 12-week intervention. (A and B) Changes in the pain scores from the baseline in the dominant and non-dominant feet, respectively, of the total subpopulation in the test group (closed bar) $(n=19)$ and placebo group (open bar) $(n=19)$; (C and D) changes in the pain scores from the baseline in the dominant and non-dominant feet, respectively, of the leading player subpopulation in the test group (closed bar) $(n=11)$ and placebo group (open bar) $(n=10)$.

chemistry and urinalysis) did not reveal any significant abnormality throughout the intervention.

\section{Discussion}

Soccer injuries have been reported to occur for the most part in the lower extremity in youth players $(17,18)$, as is the case for professional (19) and senior players $(20,21)$. The possible explanations for this are as follows: i) in soccer players, the lower extremity joints are subjected to severe mechanical stresses and are easily injured due to excessive and repetitive use of the joints; and ii) soccer players expose their lower extremity joints to repetitive intense impacts and torsional and/or axial loads. Such joint injuries may cause pain and other symptoms located in the afflicted joints.

With regard to the comparison of subtle joint pain presumably associated with minute injuries among 4 various joints (ankle, knee, hip and shoulder joints), the highest VAS scores were observed in the ankle joint, followed by the knee, hip or shoulder joint for all of the 3 pain subscales. This could be explained by the finding that relatively more sprains are localized in the ankle joint of soccer players (22). Furthermore, a substantial number of preceding studies have demonstrated that among all joint injuries occurring in soccer players the ankle sprain is the most common (17-19,23-29). The results from the present study showed that the VAS scores for the 'pain on moving' subscale were substantially higher than the scores for the 'pain at rest' subscale and 'pain on pressing' at the ankle joint, as well as other lower extremity joints in youth soccer players. This suggests that VAS scores for the 'pain on moving' subscale may be utilized as the most sensitive parameter for detecting subtle joint pain and evaluating the effect of joint-protecting treatments.

In this prospective, placebo-controlled study, in which the ankle joint was used as the target, the test product exhibited a pain-relieving effect on the ankle of the dominant foot in the subjects, particularly leading players. The pain scores were significantly reduced from the baseline in the total subpopulation and the leading player subpopulation of the test groups at week 4 and at weeks 4 and 12, respectively (Table II). Moreover, the pain score reduced significantly for the dominant feet of the leading players in the test group, compared with those in the placebo group at week 12 (Fig. 2C). By contrast, the pain scores were not significantly changed in the non-dominant feet of both the total and leading player subpopulations in the test group, as well as the dominant and non-dominant feet of the total and leading player subpopulations in the placebo group (Table II and Fig. 2). Although there is no explanation for these results, it may be speculated that the ankle joint of the dominant foot in the leading players may be subjected to increased opportunities of suffering injuries compared to the ankle joint of the opposite (non-dominant) foot. Thus, the test product may exhibit a pain-relieving effect on the ankle joint of the dominant foot in the leading player subpopulation.

Glucosamine is sometimes used by soccer players for preventing OA or relieving joint pain. However, there is currently no evidence indicating such efficacy in healthy athletes. Regarding the CCE supplement, we previously demonstrated in a clinical study that the CCE supplement has a pain-relieving effect on middle-aged and older patients with knee OA (16). Thus, it is likely that the pain-relieving effect could be extrapolated to healthy young athletes experiencing subclinical pains at the afflicted joints.

There are as yet no data available for explaining the mechanism by which the CCE supplement can alleviate joint pain in collegiate soccer players, as observed in the present study. HA, a presumable bioactive constituent of the CCE supplement, has been reported to display anti-inflammatory activities through an inhibition of the production of several pro-inflammatory cytokines (e.g., interleukin-1 $\beta$ ) or inflammatory mediators (e.g., Prostaglandin $\left.\mathrm{E}_{2}\right)(30,31)$. These observations indicate a that HA may be implicated in the favorable effect of the CCE supplement by suppressing inflammation of the affected joints, thereby relieving joint pain.

\section{References}

1. Yoshimura M, Sakamoto K, Tsuruta A, et al: Evaluation of the effect of glucosamine administration on biomarkers for cartilage and bone metabolism in soccer players. Int J Mol Med 24: 487-494, 2009.

2. Levy AS, Lohnes J, Sculley S, LeCroy M and Garrett W Chondral delamination of the knee in soccer players. Am J Sports Med 24: 634-639, 1996.

3. Buckwalter JA and Lane NE: Athletics and osteoarthritis. Am J Sports Med 25: 873-881, 1997. 
4. Gelber AC, Hochberg MC, Mead LA, Wang NY, Wigley FM and Klag MJ: Joint injury in young adults and risk for subsequent knee and hip osteoarthritis. Ann Intern Med 133: 321-328, 2000

5. Boyer T, Delaire M, Beranek L, Lasserre PP, Takeya M and Kahn MF: Incidence of previous engagement in sports among patients with symptomatic arthrosis. Controlled study. Rev Rhum Mal Osteoartic 48: 793-797, 1981.

6. Kujala UM, Kaprio J and Sarna S: Osteoarthritis of weight bearing joints of lower limbs in former élite male athletes. BMJ 22: 231-234, 1994.

7. Roos H, Lindberg H, Gärdsell P, Lohmander LS and Wingstrand $\mathrm{H}$ : The prevalence of gonarthrosis and its relation to meniscectomy in former soccer players. Am J Sports Med 22: 219-222, 1994

8. Kujala UM, Kettunen J, Paananen H, et al: Knee osteoarthritis in former runners, soccer players, weight lifters, and shooters Arthritis Rheum 38: 539-546, 1995.

9. Spector TD, Harris PA, Hart DJ, et al: Risk of osteoarthritis associated with long-term weight-bearing sports: a radiologic survey of the hips and knees in female ex-athletes and population controls. Arthritis Rheum 39: 988-995, 1996.

10. Rannou F, Poiraudeau S and Revel M: Cartilage: from biomechanics to physical therapy. Ann Readapt Med Phys 44: 259-267, 2001.

11. Lequesne M: Sport and osteoarthritis of the members. Sci Sports 19: 281-285, 2004.

12. Schwenk TL and Costley CD: When food becomes a drug: nonanabolic nutritional supplement use in athletes. Am J Sports Med 30: 907-916, 2002

13. Gorsline RT and Kaeding CC: The use of NSAIDs and nutritional supplements in athletes with osteoarthritis: prevalence, benefits, and consequences. Clin Sports Med 24: 71-82, 2005.

14. Hespel P, Maughan RJ and Greenhaff PL: Dietary supplements for football. J Sports Sci 24: 749-761, 2006.

15. Ostojic SM, Arsic M, Prodanovic S, Vukovic J and Zlatanovic M: Glucosamine administration in athletes: effects on recovery of acute knee injury. Res Sports Med 15: 113-124, 2007.

16. Nagaoka I, Nabeshima K, Murakami S, et al: Evaluation of the effects of a supplementary diet containing chicken comb extract on symptoms and cartilage metabolism in patients with knee osteoarthritis. Exp Ther Med 1: 817-827, 2010.
17. Nilsson S and Roaas A: Soccer injuries in adolescents. Am J Sports Med 6: 358-361, 1978

18. Sullivan JA, Gross RH, Grana WA and Garcia-Moral CA Evaluation of injuries in youth soccer. Am J Sports Med 8: 325-327, 1980 .

19. Albert M: Descriptive three-year data study of outdoor and indoor professional soccer injuries. J Athl Train 18: 218-220, 1983.

20. Ekstrand $\mathbf{J}$ and Gillguist J: Soccer injuries and their mechanisms: a prospective study. Med Sci Sport Exerc 15: 267-270, 1983

21. Ekstrand J and Gillquist J: The avoidability of soccer injuries. Int J Sports Med 4: 124-128, 1983.

22. Inklaar H, Bol E, Schmikli SL and Mosterd WL: Injuries in male soccer players: team risk analysis. Int J Sports Med 17: 229-234, 1996.

23. McMaster WC and Walter M: Injuries in soccer. Am J Sports Med 6: 354-357, 1978.

24. Kristensen B and Andersen C: Soccer injuries. II. The socioeconomic importance of injuries treated outside hospitals. Ugeskr Leager 141: 2703-2704, 1979.

25. Maehlum S and Daljord OA: Football injuries in Oslo: a one-year study. Br J Sports Med 18: 186-190, 1984.

26. Schmidt-Olsen S, Bünemann LK, Lade V and Brassøe JO: Soccer injuries of youth. Int J Sports Med 19: 161-164, 1985.

27. Sadat-Ali M and Sankaran-Kutty M: Soccer injuries in Saudi Arabia. Am J Sports Med 15: 500-502, 1987.

28. Keller CS, Noyes FR and Buncher CR: The medical aspects of soccer injury. Am J Sports Med 15: 230-237, 1987.

29. Høy K, Lindblad BE, Terkelsen CJ, Helleland HE and Terkelsen CJ: European soccer injuries. A prospective epidemiologic and socioeconomic study. Am J Sports Med 20: 318-322, 1992.

30. Yasui T, Akatsuka M, Tobetto K, Hayaishi M and Ando T: The effect of hyaluronan on interleukin-1 $\alpha$-induced Prostaglandin $\mathrm{E}_{2}$ production in human osteoarthritic synovial cells. Agents Actions 37: 155-156, 1992.

31. Takahashi K, Goomer RS, Harwood F, Kubo T, Hirasawa Y and Amiel D: The effects of hyaluronan on matrix metalloproteinase-3 (MMP-3), interleukin-1 $\beta$ (IL-1 $\beta$ ), and tissue inhibitor of metalloproteinase-1 (TIMP-1) gene expression during the development of osteoarthritis. Osteoarthritis Cartilage 7: 182-190, 1999. 